The Shape of Knowledge

\title{
How can I help you? User instructions in telephone calls
}

\author{
Michaël Steehouder \\ Daniël Hartman \\ University of Twente, Enschede, The Netherlands \\ m.f.steehouder@utwente.nl
}

\begin{abstract}
We a small corpus of instructions given in phone calls to customers who need support for programming their universal remote control, to make it suitable for their particular TV set, VCR, Audio, etc. Typically, in these calls the operator or 'agent' coaches the client while the client is performing actions with the equipment (turning it on, pressing buttons and codes, directing it towards the $T V$, etc.). We compared these oral instructions with the concept of a 'streamlined step procedure' [1] and other principles that are well-known from the literature about written instructions. Our conclusion is that many problems arise because the operator does not provide 'metacommunication' about the goals that have to be achieved, and because the feedback given by the client is neglected or misinterpreted.
\end{abstract}

\section{Introduction}

Although technical communicators consider it to be their mission to offer optimally designed and clear written instructions, documentation and online help, many users of technical products prefer other forms of instruction: a little help from a friend, personal assistance by a service employee, or a telephone call to a helpdesk. Several reasons may explain this preference.

- Unlike technical documentation, personal help can be tailored to the specific needs and situation of the helpseeker. For instance, the user guide of a graphic editor can explain how to change the hue or the saturation of a given picture, but a personal advisor can help us better if we want to have our relatives on the photo to have exactly that healthy blush that makes them so attractive.

- The most important reason for seeking personal help instead of using documentation is that documentation often does not contain the information that is needed. For instance, a mysterious and threatening error mes- sage can cause users to pick up the telephone and ring the helpdesk, especially if there is no information in the online help.

- Another important argument might be that personal help enables users to formulate their problems in their own terminology and language, thus avoiding the burden of finding the right entries in the index. Previous research has suggested that only $40 \%$ of the search attempts by users of software programs are successful, and that most of the failures are due to the fact that the index does not contain the entry the user has in mind.

- An important advantage of personal help may be the interactivity of the communication. The situation can often be characterized as a form of coaching, where the help-provider can give feedback to the helpseeker, can repair incorrect operations by the user, can give a warning whenever the user risks making an error, and can give extra advice whenever the user can do something more properly.

- And, finally, users may value the interpersonal approach of personal help above the impersonal, instrumental approach of online help. Talking with people is more pleasant than consulting technical documentation.

We were surprised to find only little research about giving oral technical support or instruction, at least compared with the extensive literature about writing and designing instructions, manuals, technical documentation and online help. Of course, there are some 'how to' books, and, indeed, some studies have been published about the telephone help desks. Muller [2], for instance, published a study of directory assistance telephone operators in a large US telecommunications company, showing how much personal knowledge these operators use to help their customers adequately, and also showing that these calls should not be regarded as simple questionanswer or problem-solution routines, but, on the contrary, that there are often complicated forms of collaboration and exchange of knowledge between help-seeker and 
help-provider.

Baker, Emmison and Firth [3] examined 50 calls made to a technical support line that offers assistance with problems in installing or running software. They investigated regularities in the call openings and found some interesting characteristics. One of their findings was a typical two or three step procedure within the narrative: the caller starts with mentioning the product, then narrows the conversation to the aspect of the product that causes the problem, and finally describes the problem in more detail. This almost didactic 'funnel' approach guarantees that the help-provider is able to frame the problem adequately.

Baker et al. found also that callers give a narrative account for the call. The do not simply ask a question, but they describe what happened, how they already attempted to solve the problem etc., thus presenting themselves as a competent user and justifying themselves for making claims on the expert's time and attention. The same pattern has been found in conversation within users groups on the internet [3]. Interesting is the observation by Baker et.al. that callers do not explicitly state that there is a problem. The typical formula of a narrative is " $x$ and $y$ but $z$ ", for instance: "I bought Office 97 ad installed it, but since then I haven't been able to print".

After the initial description of the problem, the helpprovider could start with giving instructions, but usually, he offers just a minimal uptake, stimulating the helpseeker to elaborate on the problem a bit more, for instance by suggesting a diagnose. Only after this elaboration, the help-provider takes over the initiative of the conversation, usually by asking specific questions that lead to a better problem diagnosis.

\section{The scope of our study}

While Baker et al. focused their study on the opening sequences, and in particular on the way the problem is stated by the help-seeker, our study focuses on the way the actual instruction is given at a later stage in the conversation. The aim of this study is comparable with the aims of Baker et.al.: we try to identify some regularities in conversation.

Our study is based on transcriptions of six calls made by users of a programmable remote control for electronic household devices (TC, Video System, etc,). All of the callers needed support to program their remote control because the appropriate code for their equipment was not pre-set on the remote control, nor was it given in the instructions for use. Although the call center is situated in Enschede, The Netherlands, calls came from all over Europe and took place in different languages: English, German, French, Spanish and Italian.

In this paper, we will use only one of these transcriptions. The call took place on 28 January 1998. The caller (c) is a male from the UK; the agent (a) is a female from The Netherlands. The help request was made to solve a problem with programming the remote control for getting teletext on a Hitachi television. The total duration of the call was 27 minutes and 59 seconds. (A more detailed analysis is published in [4]

\section{The opening of the call}

Although our main purpose is not to examine the opening sequences of the call, it is interesting to see that the client

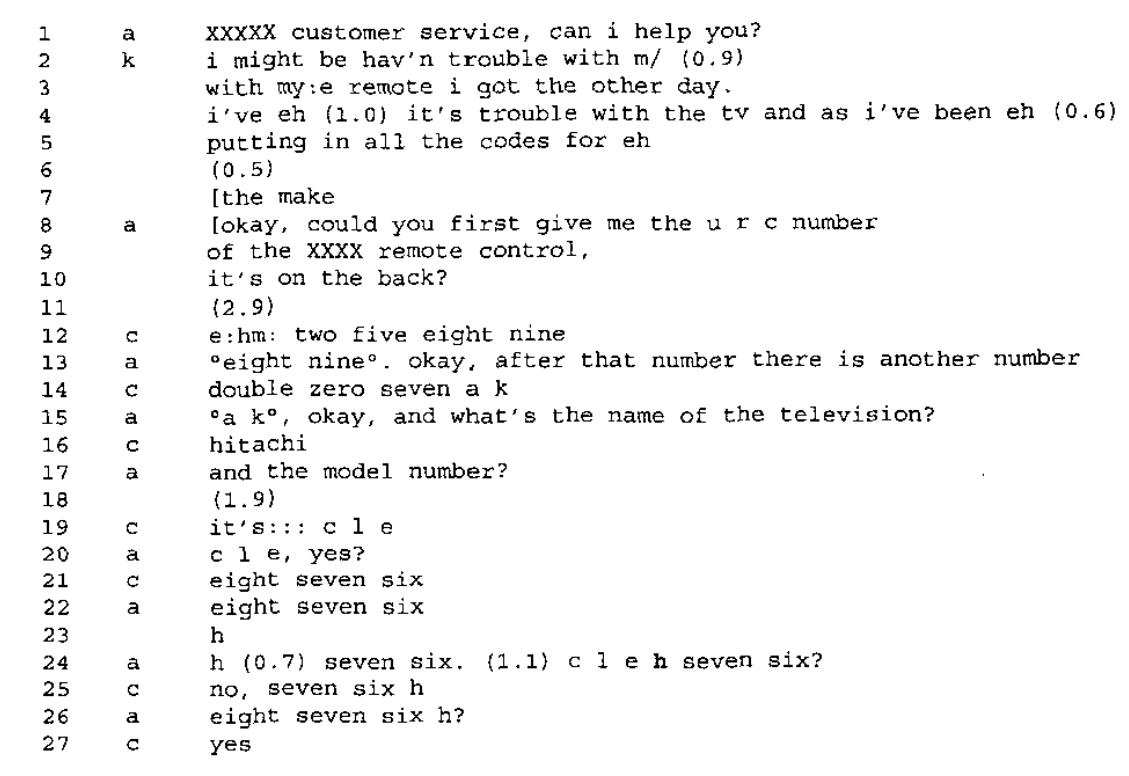


The Shape of Knowledge

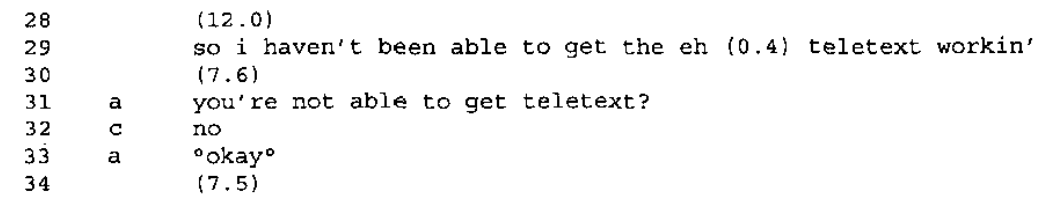

wants to start with a 'funnel' narrative as identified by Baker et al. [3] (line 3-6). However, he is interrupted by the agent who seems to need some preliminary information (line 8-27). It seems that the interruption in line 8 has been interpreted by the client as a signal that the agent wants to take over the control of the conversation. Only after a long pause of 12 seconds, the help-seeker takes the opportunity to resume the narrative (line 29), but the long pause in line 30 suggests that he expects the agent to take over again - which she does in line 31 with what at this stage may be seen as a thetorical question. (Rhetorical questions are, according to Dillon [4] a means of establishing an superior/authoritative position towards the listener - so this question seems to confirm the agent's control over the conversation).

\section{Getting control over the caller's actions}

Much of the time in the call is taken up by instructions for pressing buttons, given by the agent. Of course, it is important that these instructions are followed accurately by the caller. A typical example of what can happen is shown in line 60-75.

This fragment shows one of the typical problems with giving stepwise instructions by telephone: the agent cannot see what the caller is actually doing, and therefore she needs a form of feedback. When she does not get a feedback signal (line 61), she repeats the instruction with some more emphasis ('please' and 'can' instead of 'could') to elicit a verbal reaction, which she gets in line 63. Feedback is given again in line 66 , and it seems that she is now confident enough that the caller is really doing what she tells him to do, since she gives the remaining ac- tions without waiting or asking for feedback (line 67-69). After line 69 the teletext is expected to be seen on the screen, and the agent seems to expect an approval from the caller. When this reaction stays away (pause in line 70 ), she seems to interpret this as a signal that the effect did not occur, and she first wonders if the caller really did what she told him (line 71), and only then she verifies explicitly whether the expected effect really did not take place (line 73). In sum, the fragment shows that eliciting feedback from the caller is an important task for the agent and that it is not easy to get it.

How important it is to get control over the caller's actions, becomes clear when we look at the continuation of the call in the 76-97 (next page). The agent imputes the failure of the procedure not to the possibility that she gave the wrong instructions (what actually was the case), but she assumes that the caller made mistakes. So, she verifies again whether he really did what she told him (line 76 , which expresses the same as line 71 above), and again she expresses her suspicion that the caller was to far away from the television (line 79 , cf. line 60 and 62 above). Than she starts the same procedure again (line 81-82). Remarkably, she does not wait for the caller's feedback here, but she presents all actions at once, formulating shorter ('once tv' in stead of 'press once the tv button'), and she forgets to mention that the last three has to be hold a little longer.

It seems that the agent is now convinced that the failure was not the caller's fault, since in line 86 she asks for one moment and she pauses 24.2 seconds to consult her (online) documentation. She does not explain this to the caller, however, underlining that she has the 'control' over the conversation.

Line 69 shows a specific problem connected with

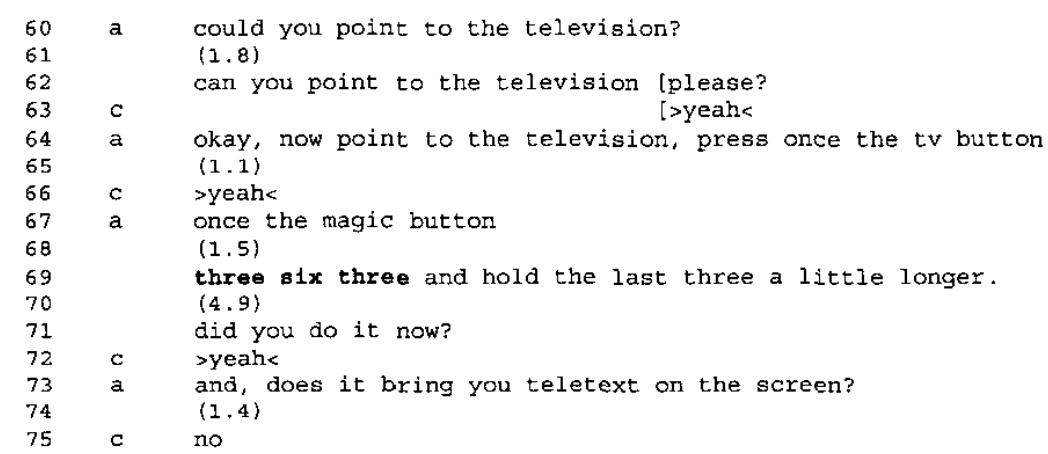


The Shape of Knowledge

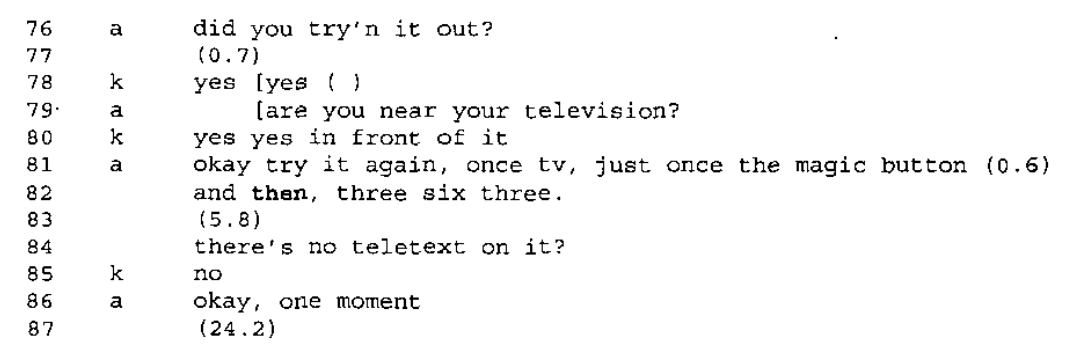

giving oral instructions: the agent has to be sure that button 3 is not released too soon, before she can say to hold it a little longer. Strange enough, this detail is left out in the repetition of the procedure (line 82 ).

In the following fragment, a little later in the call, we notice that the agent tries to prevent an error with a simi- lar procedure by splitting up the procedure, using a different sequential order of the information, and stressing the word hold (line 98-99). Note the interesting pause and emphasis in line 97 ('correctly'); apparently the agent is still not convinced that the caller really points to the television.

$$
\begin{aligned}
& \text { just }(0.7) \text { point co:rrectly to the television, } \\
& 98 \text { just once tv, once magic, four two, }
\end{aligned}
$$

There are many moments later in the call where the same issue returns. In one case she stresses the importance very explicitly (line 264-367).

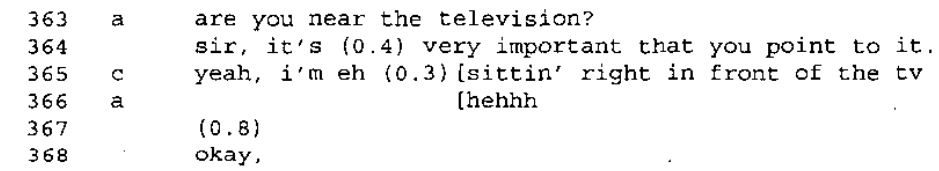

How important it is for the agent to have control of the caller's action, is illustrated again in line 512-548, much later in the call. The almost impolite 'It's very important that you do what I'm telling you' (line 518) is followed by a strict step by step instruction, spoken aloud and with emphasis, and responded to by the caller's explicit confirmations, that show that he 'takes over' this commandmode conversation.

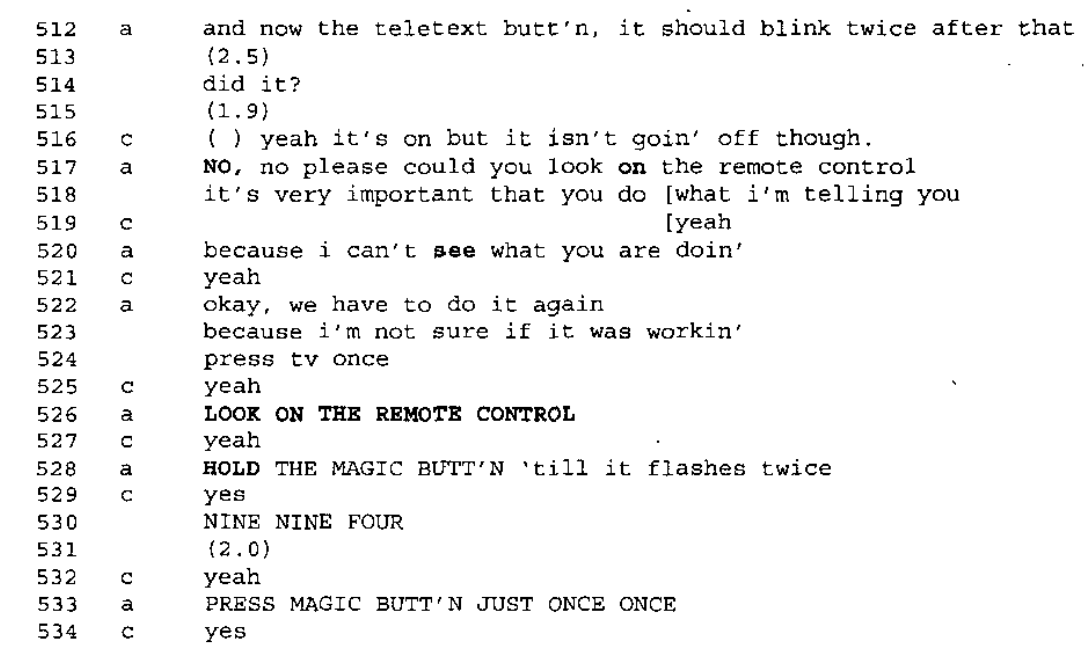


The Shape of Knowledge

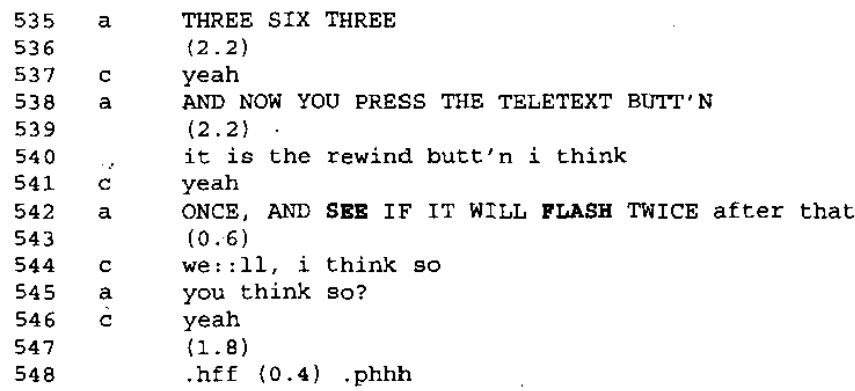

In sum, we identified the following strategies of getting control over the actions of the caller:

- insert short pauses after each action to indicate that the caller has to do what has been told, and to give the opportunity to confirm that (yeah, ok);

- ask for explicit feedback and/or asking whether the caller really did what he was expected to do;

- split up sequences

- emphasize crucial steps or elements in steps (such as hold in line 99 and the instructions in line 526-542)

- tell the caller how important it is to follow the directions.

\section{Making sense of the procedures}

When overlooking the protocol it stands out that the agent restricts herself to giving instructions on a very low level: she seems not to try to explain to the caller what is going on, or what she is thinking of doing. Steehouder, Karreman \& Ummelen [6], based on a review of research literature, pointed out that readers of instructions could benefit from 'sense making features' that can make instructions more meaningful. They mention in particular:

- Creating hierarchy in sequences of instructions, especially by using the distinction between a functional and a syntactic level.

- Relating the sequence of steps to real-life goals and situations.

- Adding declarative information to instructions.

The examples above show that the agent is not creating a sense of hierarchy in her instructions. She never refers to higher level goals in the series of actions she is referring to. The oral instruction lacks the equivalents of common features in 'streamlined step procedures' [1] such as titles and subtitles that establish goals and sub goals, or conceptual elements that explain why steps are important, or what a step really means for the functioning of the device.

One possible explanation is that the agent overestimates the expertise of the caller. She might have been mislead by the fact that the caller started with describing the problem in a manner that suggests that he understands how programming the remote control works - he might have learned that from the user instructions. The dialogue in line 35-55 shows that the caller is assumed to know how to program in the code, and to understand what the 'normal functions' are.

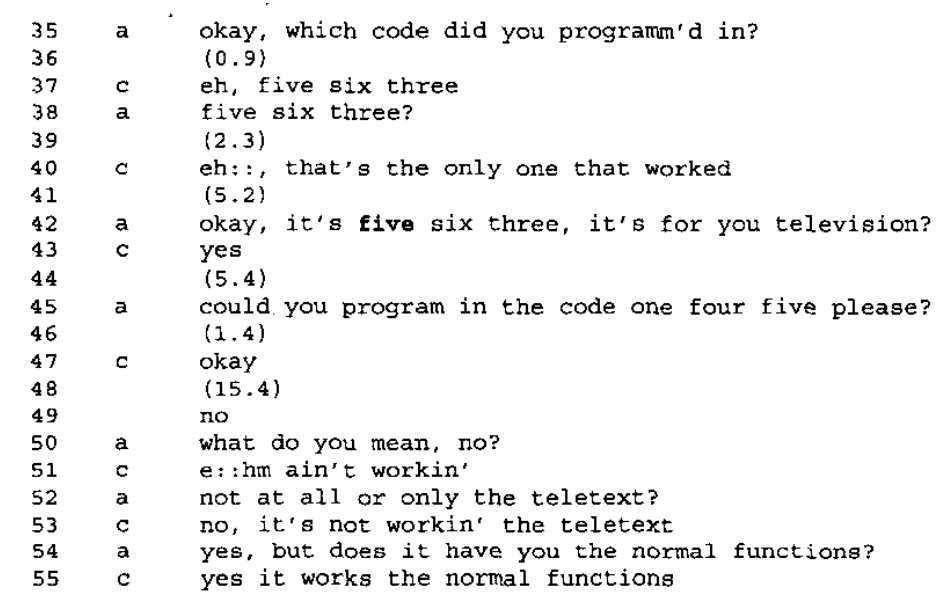


Line 40 suggest that the caller has tried out other possibilities, and line 43 suggests that he understands what he is doing very well. Consequently, the first direction in line 45 is given on a relatively high level of actions. In fact Program in is a procedure that consists of the following steps:

1 Turn your TV on

2 Press the TV-button on the remote control once

3 Press the MAGIC-button on the remote control until the Red light blinks twice

4 Insert the code using the numerical keys (the red light will blink twice)

5 Point the remote control to the TV and press On/Off

6 If your TV stops, the TV is adjusted to the new code

This procedures is explained in the user instructions, which may have been read by the caller. The caller's 'okay' (line 47), and the subsequent long pause (line 48) confirm that the caller can be addressed to on this level of expertise. Another confirmation can be found in line 5455 where the caller turns out to be able to verify what works and what does not.

However, the caller's high level of expertise is not recognized in the following of the call, since the agent never explains what she thinks that happens, nor does she explain how she is trying to solve the problem. It seems that the caller is put into the role of an 'operator' [7], who only has to follow simple directions.

The caller, on the other hand, seems to adopt this role at first without problems, possibly believing that this is the best way to solve the problem as soon and easily as possible. He does not show any initiative, nor does he come up with suggestions. And in the exceptional moment where he tries to give some more information than simply yes or no, the agent muzzles him (line 113-127):

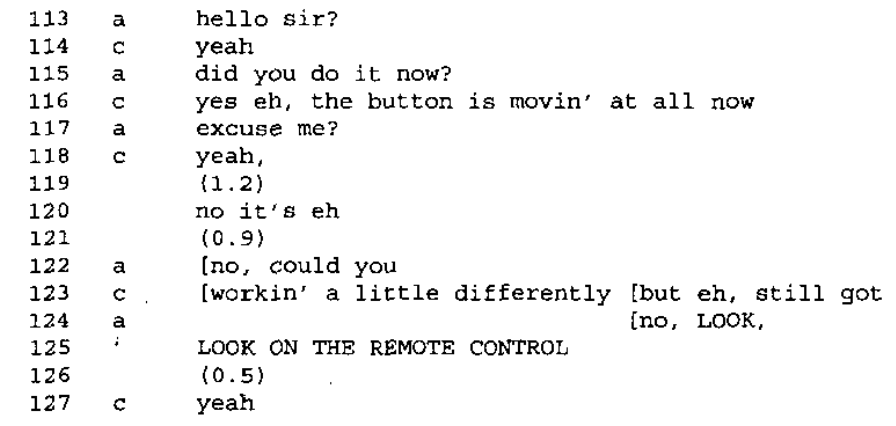

Only much later in the call, after endless useless attempts to get the teletext working and long pauses, and after a confession of the agent that she reaches the limits of her abilities (line 370), the caller shows some initiative again,

by asserting that the situation seems hopeless (line 377 . 382). This mutual admission seems to be a turning point in the conversation. Shortly after it, the agent explains for the first time what she is going to do:

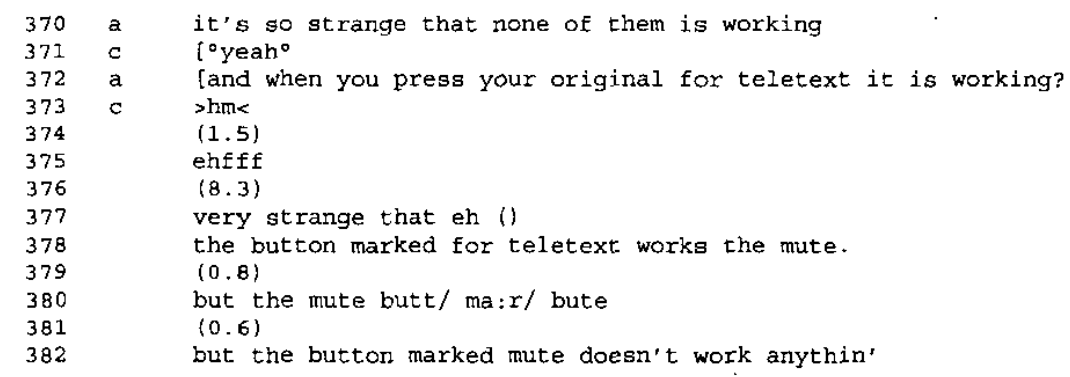

From now on, she is now and then involving the caller in the problem solving process by asking more background information and especially by indicating more often what her plans are: 
The Shape of Knowledge

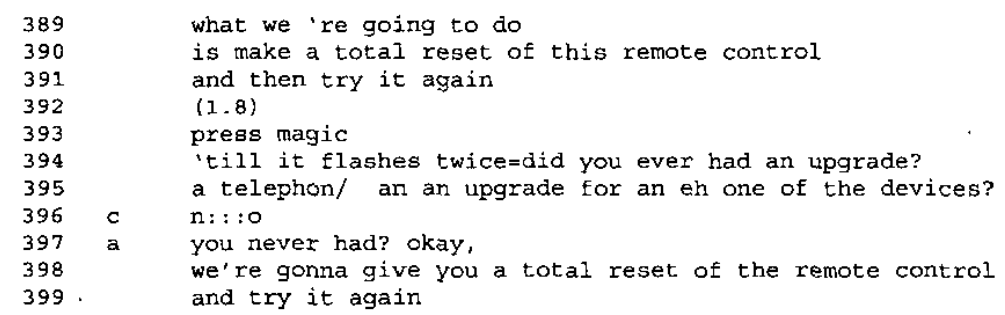

The following examples show again how the agent tries to explain what she is doing. Line 496 in particular suggests that the agent is now more often choosing the caller's per- spective. She seems to suspect that the caller believes that he has to point to the television, and she corrects this assumption.

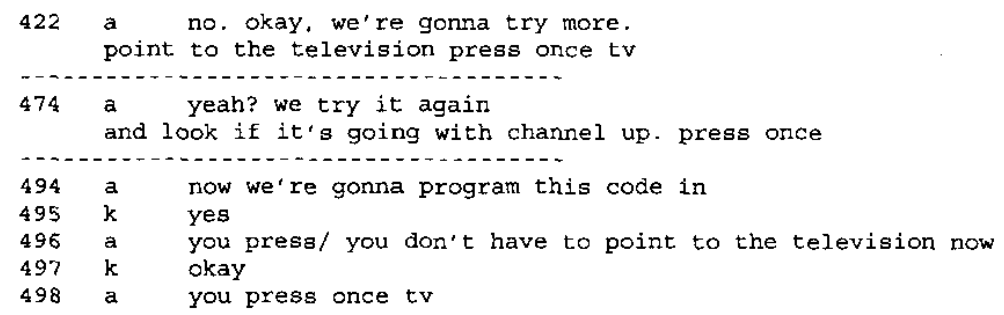

\section{Success at last}

Surprisingly enough, after so many failures and pauses, the problem is solved at the end of the call, because the agent realizes that she did not give the right instruction. It seems that this could have happened earlier if she had listened more carefully to the signals given by the caller. However, we cannot be sure of that from this particular protocol.

\section{Conclusions}

Our analysis has shown that giving step-by-step instructions via telephone is a complicated task. The protocol we used for our analysis is not exceptional - the five other protocols that we collected show similar problems and strategies by the agents.

We noticed in all protocols the strong efforts that agents have to make to control the behavior of the caller: making the caller follow the directions exactly, immediately, accurately, and getting enough feedback to know what the effect is.

We also found that agents try to give instructions on the low syntactical level, confining themselves to the syntactical level (the buttons) without explaining the goals and sub goals, their line of thought, or the working of the device. They put the caller in the role of passive operator, and they put themselves in the role of director.

We found in all protocols that the callers accepted the role of 'passive operator' without any protest. This is somewhat in contrast to the findings of Muller (1999) who found much more 'collaborative problem solving'; however, this difference is easily explained by the nontechnical character of Muller's calls, and the more technical content of ours. Apparently, callers to a technical support center as ours are only interested in a quick and easy solution for their problem and are not eager to 'make sense' of it. However, the painful and time-consuming sessions, with so many tries and retries of procedures suggest that another approach might be more effective in the end. But we naturally cannot prove that.

We noticed, finally, that the calls were generally very impersonal. Apart from some obligatory friendliness in the beginning ('How can I help you'), we found only occasionally signals of empathy, hopelessness or other emotions.

It is too early to draw practical conclusions from our observations. Our material consists only of a small collection of protocols, and only two were analyzed in full detail. Moreover, the remote controls that were the subject of these calls, form a very specific type of technical device, and their characteristic features will inevitably have influenced the course of the conversation. Moreover, there is no evidence that another approach, for instance with more 'sense making', would lead to shorter and more successful calls, nor that it would lead to a higher satisfaction of customers. But, on the other hand, we think that our analysis form a useful starting pint form more research into the effectiveness of personal oral instructions for users of technical appliances. 


\section{References}

[1] Farkas, D. K., The logical and rhetorical construction of procedural discourse. Technical communication 46(I): 42-54, 1999.

[2] Baker, C., M. Emmison and A. Firth, Discovering order in opening sequences, calls to a software helpline. In: A. McHoul, and M. Rapley (Eds.) How to analyse talk in institutional settings. $A$ casebook of methods (pp. 41-56). Continuum, Londing, 2001.

[3] Hartman, D. 'Je moet doen wat ik zeg'. ['Do as I say']. Ma Thesis, University of Twente, 2003.

[4] Dillon, G. Rhetoric as social imagination. Explorations in the interpersonal function of language. Indiana University Press, Bloomington IN, 1986.

[5] Muller, M. J. Invisible work of telephone operators: an ethnocritical analysis. Computer Supported Cooperative Work 8: 31-61, 1999.

[6] Steehouder, M., Author and reader in instructions for use. In Crossroads in communication. Proceedings IPCC 97. (pp. 77-90). IEEE, Piscataway, NJ, 1997.

[7] Steehouder, M., J. Karreman and N. Ummelen, Making sense of step-by-step procedures. In Technology \& Teamwork. IPCC 2000. Cambridge, Massachusetts (pp. 463-475). IEEE, Piscataway, 2000.

[8] Joosten, A. Psychosociale verklaringen voor klachten in huisarts-patiëntgesprekken. $\mathrm{PhD}$ thesis, University of Groningen, 1997

\section{About the Authors}

Michaël Steehouder holds the chair of Technical Communication at the University of Twente. He is also chair of the Dutch society for Technical Communication STIC and board member of TCeurope. His research interests include the design of government forms and user instruction, and rhetorical aspects of technical communication. He is an Associate Editor of IEEE Transactions on Professional Communication.

Daniël Hartman has a MSc in Philosophy of Technology at the University of Twente. After working for Universal Electronics, he became a staff member of the association of victims of the Enschede Firework Explosion of 2001.

\section{Appendix 1: Explanation of the transcription codes}

The coding conventions are adopted from [8]

\begin{tabular}{|c|c|}
\hline 31 & line number \\
\hline$a, c$ & agent, caller \\
\hline (.) & pause shorter than 0.3 seconds \\
\hline$(1,5)$ & pause in seconds \\
\hline . , ? & $\begin{array}{l}\text { decreasing, increasing or questioning tone at } \\
\text { the end of an utterance }\end{array}$ \\
\hline$\uparrow \downarrow$ & $\begin{array}{l}\text { decreasing, increasing intonation in the next } \\
\text { word }\end{array}$ \\
\hline $\begin{array}{l}\text { wo }[\text { rd } \\
\quad \text { Iword } \\
=\end{array}$ & $\begin{array}{l}\text { Utterances of two speakers spoken simulta- } \\
\text { neously } \\
\text { immediate following of two utterances }\end{array}$ \\
\hline wo/ & broken off \\
\hline wo: $x d$ & $\begin{array}{l}\text { prolongation of vowel or consonant: the } \\
\text { more dots, the longer the pronunciation }\end{array}$ \\
\hline WORD & spoken aloud \\
\hline word & spoken with emphasis \\
\hline${ }^{\circ}$ word' & spoken quietly \\
\hline .ghh/.thh & inhalation by mouth \\
\hline fhr & inhalation by nose \\
\hline .hh & sigh \\
\hline$>$ word< & fast \\
\hline <word> & slow \\
\hline 1$)$ & not understood \\
\hline$\{$ word $\}$ & spoken inarticulately \\
\hline
\end{tabular}

